\title{
Impaired myocardial performance in a normotensive rat model of intrauterine growth restriction
}

\author{
Carlos Menendez-Castro', Okan Toka ${ }^{2}$, Fabian Fahlbusch', Nada Cordasic ${ }^{3}$, Rainer Wachtveitl', Karl F. Hilgers ${ }^{3}$, \\ Wolfgang Rascher ${ }^{1}$ and Andrea Hartner ${ }^{1}$
}

\begin{abstract}
BACKGROUND: Intrauterine growth restriction (IUGR) is an important risk factor for cardiovascular disease. Previous studies revealed altered myocardial matrix composition after IUGR. We hypothesized that IUGR is accompanied by compromised myocardial performance independently from arterial hypertension.
\end{abstract}

METHODS: IUGR was induced in Wistar rats by maternal protein restriction, and hearts of male offspring were studied using echocardiography, immunohistochemistry, real-time $P C R$, and western blot analysis.

RESULTS: At day 70 of life, in the absence of arterial hypertension (mean arterial blood pressure: $101.3 \pm 7.1 \mathrm{mmHg}$ in IUGR vs. $105.3 \pm 4.6 \mathrm{mmHg}$ in controls, not significant (NS)), echocardiography showed a reduced contractility (ejection fraction: $65.4 \pm 1.8 \%$ in IUGR vs. $82.2 \pm 1.5 \%$ in controls, $P<0.001$ ) of a more distensible myocardium in IUGR rats. Altered expression patterns of myosin chains and titin isoforms and increased expression levels of atrial natriuretic peptide, Na/K-ATPase, and $\beta$-adrenergic receptor 1 were detected. A higher number of cardiac fibroblasts and vascular cross-sections were observed in IUGR rats, accompanied by elevated expression of hypoxia inducible factor 1 target genes, such as vascular endothelial growth factor and its receptors.

CONCLUSION: We observed a blood pressure-independent impairment of myocardial function after IUGR, which possibly favors cardiovascular disease later in life. Some IUGR-induced myocardial changes (e.g., sarcomeric components) may partly explain the compromised cardiac performance, whereas others (e.g., elevated vascular supply) reflect compensatory mechanisms.

ntrauterine growth restriction (IUGR) is associated with a higher incidence of arterial hypertension, type 2 diabetes, and hyperlipidemia in adulthood and was identified as a relevant risk factor for cardiovascular disease by several epidemiologic studies (1). The pathogenetic link between fetal deficiency and cardiovascular disease later in life remains unclear. The development of a metabolic syndrome, which is often seen in IUGR individuals, might secondarily lead to cardiovascular dysfunction (2). Alternatively, structural and functional cardiovascular changes might reflect primary IUGR-induced events during organogenesis. Supporting this notion, Barker (3) developed the hypothesis of "fetal programming," which postulates that the adverse intrauterine environment impairs fetal growth and differentiation which in turn leads to a lifelong alteration of cardiovascular structure and integrity.

In this context, it could be shown that IUGR is associated with a reduced number of cardiomyocytes at the time of birth (4). Moreover, fetal hypoxia, a frequent cause of IUGR, resulted in early alterations of myocardial vasculature and changes in cardiac structure and function resembling dilated cardiomyopathy in chicks (5). The idea of early and primary cardiac changes after IUGR is supported by recent clinical studies. Crispi et al. (6) showed that overt changes of myocardial structure and functionality occurred in IUGR patients already in childhood. There are, however, conflicting reports on myocardial functionality in rat models of IUGR: Lim et al. (7) detected cardiac hypertrophy without impairment of myocardial performance in adult individuals after IUGR, while Cheema et al. (8) described a reduced cardiac output in juvenile IUGR animals.

IUGR is induced by a variety of different pathogenetic factors such as nutritional deficiency and reduced placental perfusion. These pathomechanisms are used in different animal models to induce IUGR (9). In this study, IUGR was induced by maternal protein restriction. According to our observations in this model, no hypertension, no hypercholesteremia, and no atherosclerosis were detected at the time points investigated (10). On the other hand, we observed a moderate increase in myocardial collagen deposition, which was, however, not accompanied by overt myocardial fibrosis or hypertrophy (10).

Based on these observations, we hypothesized that IUGR leads to a decline in myocardial performance and that this is accompanied by a dysregulation of contractile components and functional parameters, which possibly favor the development of cardiac disease later in life. 


\section{RESULTS}

As reported previously (10), male offspring of dams fed a low protein diet during pregnancy (LP rats (rats with IUGR)) showed significantly lower birth weights $(4.90 \pm 0.5 \mathrm{~g}$ in LP vs. $6.39 \pm 0.34 \mathrm{~g}$ in NP rats (control rats), $P<0.001$ ). Body weights remained reduced in LP animals until day 70 of life $(365.8 \pm 6.6 \mathrm{~g}$ in LP rats vs. $383.7 \pm 4.4 \mathrm{~g}$ in NP rats, $P<0.05)$. The assessment of relative heart weights at this time point showed no significant differences in LP and NP animals, confirming the absence of myocardial hypertrophy $(4.40 \pm 0.40 \mathrm{mg} / \mathrm{g}$ in LP rats vs. $4.74 \pm 0.34 \mathrm{mg} / \mathrm{g}$ in NP rats, NS). Mean arterial blood pressure values, measured by intraarterial catheters, showed no differences in LP and NP rats at day $70(101.3 \pm 7.1 \mathrm{mmHg}$ in LP rats vs. $105.3 \pm 4.6 \mathrm{mmHg}$ in NP rats, NS).

\section{Echocardiography}

We performed echocardiography in 70-d-old animals. Heart rate was comparable between NP and LP rats $(333.8 \pm 9.43 \mathrm{bpm}$ in NP rats vs. $320.7 \pm 9.70 \mathrm{bpm}$ in LP rats, NS). We detected significantly reduced values for ejection fraction (Figure 1a) and consecutively for fractional shortening $(31.8 \pm 0.70 \%$ in LP rats vs. $43.6 \pm 1.96 \%$ in NP rats, $P<0.001)$ in LP rats. Assessing left ventricular anteriolateral wall thickness, we also obtained significantly reduced values in LP animals (Figure 1b), whereas no significant difference of left ventricular posterior
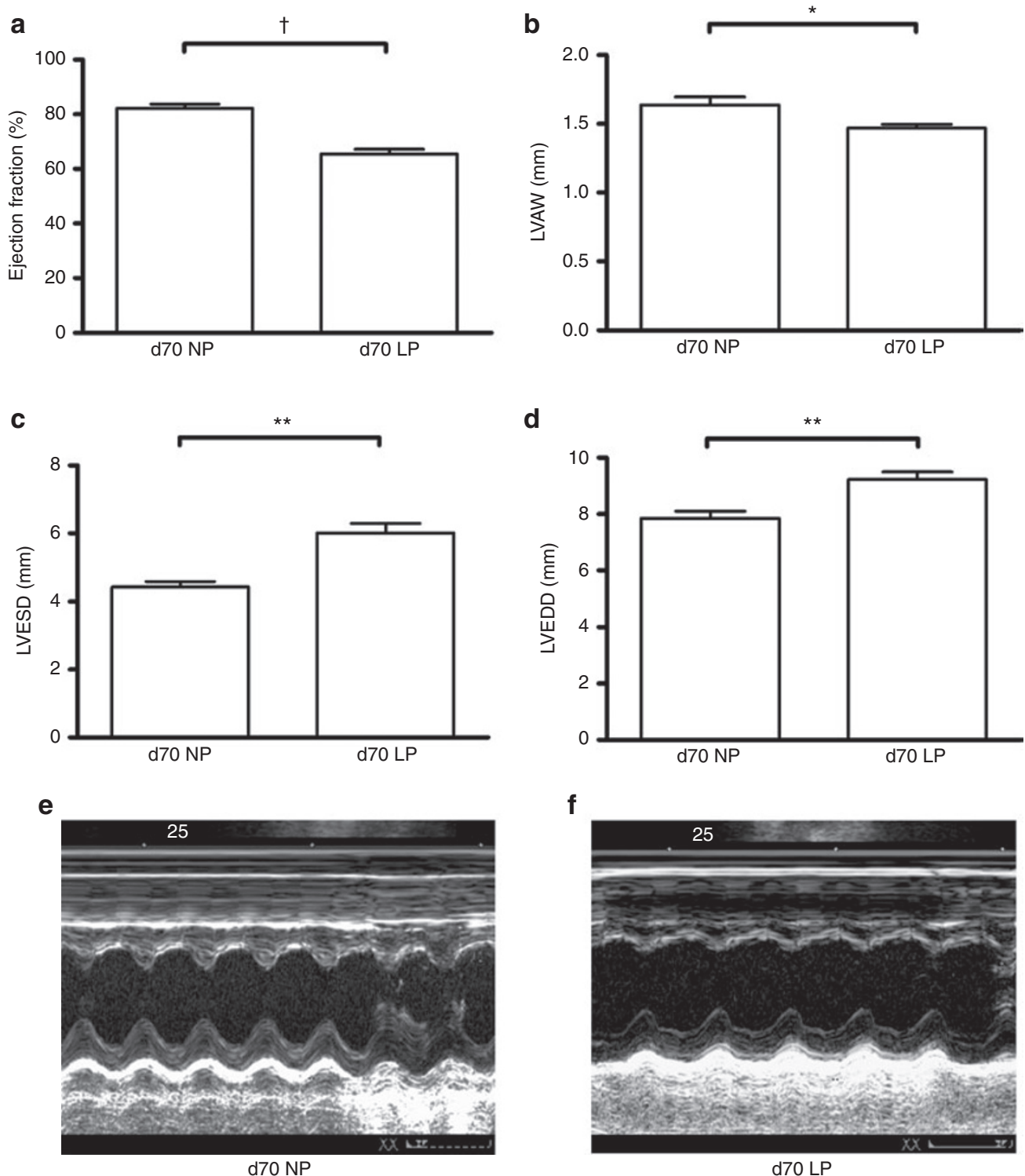

Figure 1. Echocardiographic parameters. (a) Ejection fraction (EF); (b) left ventricular anterior wall thickness (LVAW); (c) left ventricular end-systolic diameter (LVESD); (d) left ventricular end-diastolic diameter (LVEDD) at day 70 (d70) of life. Cardiac echocardiograms were performed on 70-d-old NP and LP animals. Shown are representative M-mode echocardiograms in which a one-dimensional signal across the center of a transverse section of the heart is visualized over time. (e) Preserved cardiac function in NP animals and (f) mild left ventricular dilation in LP animals could be detected. Particularly left ventricular anterior wall contractility seems to be impaired in LP animals. Both images are shown with equal magnitude (see dot scale). Data are means \pm SEM. ${ }^{*} P<0.05,{ }^{* *} P<0.01,{ }^{\dagger} P<0.001$. LP, rats with intrauterine growth restriction; NP, control rats. 
wall thickness could be detected $(1.45 \pm 0.05 \mathrm{~mm}$ in LP vs. $1.58 \pm 0.07 \mathrm{~mm}$ in NP rats, NS). Left ventricular end-systolic diameter (LVESD) and left ventricular end-diastolic diameter (LVEDD) were augmented in LP rats (Figure $1 \mathbf{c}, \mathbf{d}$ ).

\section{Parameters of Myocardial Structure and Functionality}

In hearts of 1-d-old LP and NP rats, we could not detect any difference of the mRNA expression of $\alpha$-myosin heavy chain $(\alpha-\mathrm{MHC})$ and $\beta$-myosin heavy chain ( $\beta$-MHC). At day 70 , we observed significantly higher mRNA levels of these contractile myocardial components in LP rats (Table 1). Evaluation of the

Table 1. mRNA expression $\alpha-M H C, \beta-M H C$, and the titin isoforms $\mathrm{N} 2 \mathrm{~B}$ and N2BA in myocardial tissue of NP and LP rats at $d 1$ and d70 of life

\begin{tabular}{|c|c|c|c|c|}
\hline & \multicolumn{2}{|c|}{ d1 } & \multicolumn{2}{|c|}{$\mathrm{d} 70$} \\
\hline & NP & LP & NP & LP \\
\hline$\alpha-\mathrm{MHC}$ (fold induction) & $1.00 \pm 0.10$ & $0.82 \pm 0.11$ & $2.23 \pm 1.24$ & $7.64 \pm 1.81^{*}$ \\
\hline$\beta-\mathrm{MHC}$ (fold induction) & $1.00 \pm 0.18$ & $1.01 \pm 0.09$ & $1.85 \pm 0.77$ & $9.33 \pm 2.41^{* *}$ \\
\hline N2B (fold induction) & $1.02 \pm 0.08$ & $1.07 \pm 0.14$ & $1.45 \pm 0.34$ & $3.28 \pm 0.31^{* *}$ \\
\hline N2BA (fold induction) & $1.02 \pm 0.17$ & $0.97 \pm 0.09$ & $0.69 \pm 0.22$ & $2.04 \pm 0.26^{* *}$ \\
\hline
\end{tabular}

expressional ratio of $\alpha-\mathrm{MHC}$ to $\beta$-MHC revealed a trend to a decreased $\alpha-\mathrm{MHC} / \beta-\mathrm{MHC}$ ratio in LP rats at day 70 of life ( $0.77 \pm 0.14$ vs. $1.15 \pm 0.21$ in NP; NS). We also assessed expression patterns of the two titin isoforms N2B and N2BA. In 1-d old LP and NP rats, expression levels of both isoforms were comparable. In 70-d-old animals, the expression of N2B and of N2BA was significantly augmented in LP animals (Table 1). Evaluation of the expressional ratio of N2BA to $\mathrm{N} 2 \mathrm{~B}$ revealed an increased $\mathrm{N} 2 \mathrm{BA} / \mathrm{N} 2 \mathrm{~B}$ ratio in LP rats $(0.66 \pm 0.05$ vs. $0.44 \pm 0.04$ in NP rats; $\mathrm{p}<0.05)$. Similarly, myocardial expression of $\beta$-adrenergic receptor 1 (Adrb-1), Na/K-ATPase, and atrial natriuretic peptide (ANP) was comparable in LP and NP rats at day 1, while at day 70 of life, we detected increased expression levels in LP animals (Figure 2a-eE).

\section{Hypoxia, Neoangiogenesis, and Vascularization}

To address the question, if hypoxia might contribute to the detected myocardial changes, we evaluated the expression of several hypoxia inducible factor 1 (HIF-1) target genes: vascular endothelial growth factor (VEGF), heme oxygenase 1 (HO-1), hypoxia inducible gene 2 (HIG-2), and insulin-like growth factor binding protein 3 (IGFBP-3) as surrogate markers of HIF-1 stabilization. Assessment of myocardial mRNA expression of the HIF-1 target genes, IGFBP-3, HO-1, and HIG-2, in NP and LP rats showed no differences at day 1 of

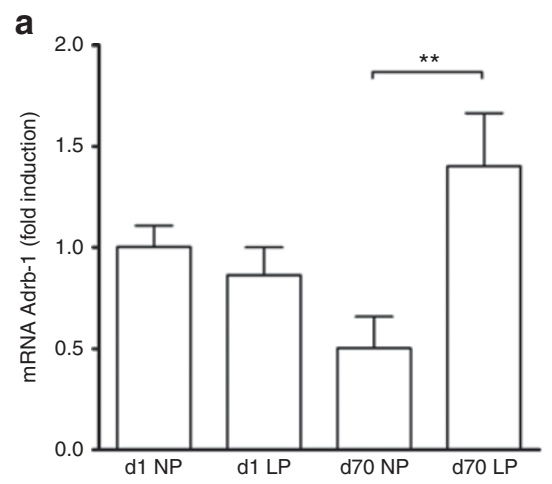

C

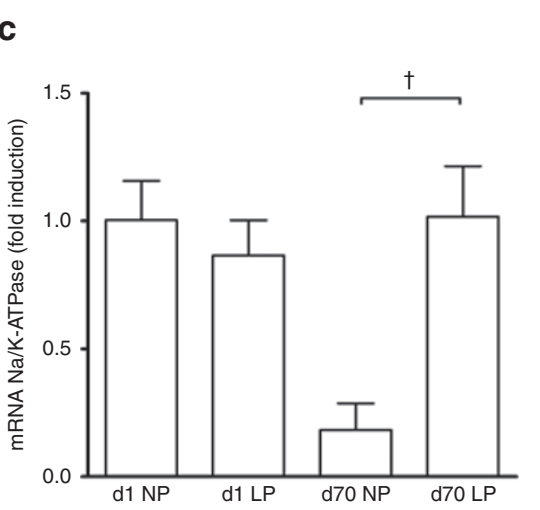

b

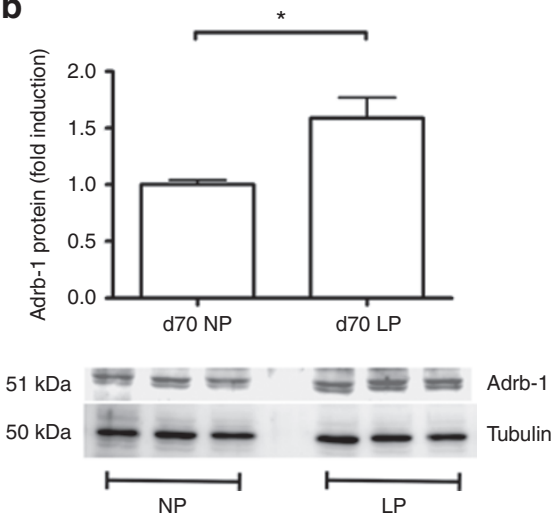

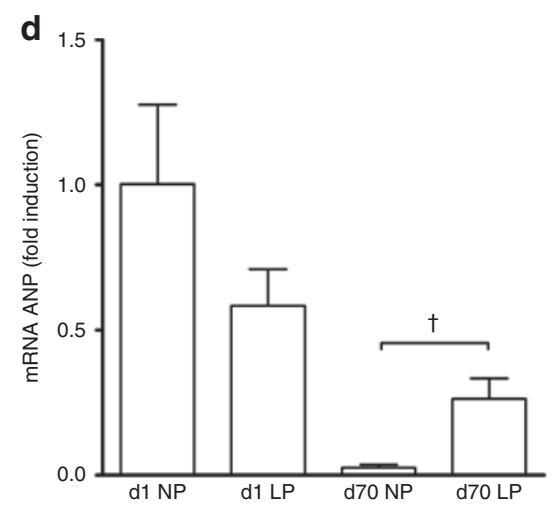

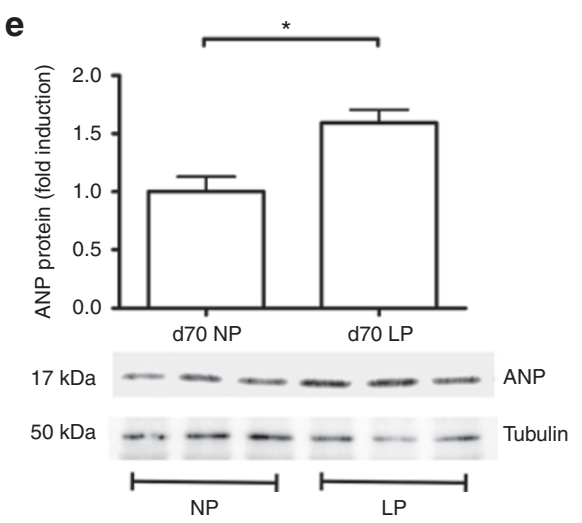

Figure 2. Myocardial expression of parameters of myocardial functionality. Expression of $\beta$-adrenergic receptor 1 (Adrb-1) (a) on the mRNA level and (b) on the protein level; (c) Na/K-ATPase on the mRNA level; and atrial natriuretic peptide (ANP) (d) on the mRNA level and (e) on the protein level in rats at day 1 (d1) and at day 70 (d70) of life. Tubulin staining served as a loading control for western blot analyses. Data are means $\pm S E M$. ${ }^{*} P<0.05,{ }^{* *} P<0.01$, ${ }^{+} P<0.001$. LP, rats with intrauterine growth restriction; NP, control rats. 


\section{Articles | Menendez-Castro et al.}

life but a significant overexpression at day 70 of life in LP animals (Table 2). In the hearts of 1-d-old LP and NP rats, mRNA levels of VEGF, neuropilin 1 (NRP1), VEGF receptor 1 (Flt1), and VEGF receptor 2 (Flk1) showed no difference. In contrast, we observed a significant and concordant overexpression of these angiogenic factors in the myocardium of LP animals at day 70 of life (Figure 3a-e).

Quantifying the vascular supply of the myocardium by rat endothelial cell antigen (RECA) staining in 1-d-old rats, we could not detect any differences between LP and NP animals (Figure 4a). At day 70 of life, LP animals, however, showed a significantly higher number of myocardial vascular crosssections (Figure $\mathbf{4 b}-\mathrm{dD}$ ). We detected increased myocardial expression of $\alpha$-smooth muscle actin ( $\alpha$-SMA) at the mRNA

Table 2. mRNA expression of HIF-1 target genes: IGFBP-3, HO-1, and HIG-2 in myocardial tissue of NP and LP rats at $d 1$ and $d 70$ of life

\begin{tabular}{|c|c|c|c|c|}
\hline & \multicolumn{2}{|c|}{ d1 } & \multicolumn{2}{|c|}{$d 70$} \\
\hline & NP & LP & NP & LP \\
\hline IGFBP-3 (fold induction) & $1.00 \pm 0.14$ & $0.77 \pm 0.11$ & $1.00 \pm 0.24$ & $10.90 \pm 3.06^{*}$ \\
\hline HO-1 (fold induction) & $1.00 \pm 0.18$ & $1.10 \pm 0.20$ & $1.00 \pm 0.49$ & $7.08 \pm 2.63^{* *}$ \\
\hline HIG-2 (fold induction) & $1.00 \pm 0.08$ & $0.83 \pm 0.11$ & $1.00 \pm 0.40$ & $5.20 \pm 1.30^{* *}$ \\
\hline
\end{tabular}

and protein level in 70-d-old LP rats (Figure 5a,b). We also observed a higher number of $\alpha$-SMA-positive vascular crosssections in 70 -d-old IUGR rats (Figure $5 \mathrm{c}-\mathrm{eE}$ ). Using double immunofluorescence for $\alpha$-SMA and RECA, we showed that $\alpha$-SMA and RECA were always localized together indicating that increased expression levels of $\alpha$-SMA could be attributed to an increased number of $\alpha$-SMA-positive arterioles in LP rats (Figure 5f).

Increased $\alpha$-SMA expression might also be a consequence of an increase in activated myofibroblasts. Therefore, we evaluated the abundance of fibroblasts: real-time PCR and western blot analyses revealed higher expression levels of the fibroblast marker vimentin in the myocardium of 70-d-old LP rats (Figure 6a,b). By immunohistochemistry, we detected a higher number of vimentin-positive fibroblasts in the myocardium of LP rats (Figure $6 \mathrm{c}-\mathrm{e}$ ). To confirm these data, we also stained for a second marker for myocardial fibroblasts, the discoidin domain receptor 2 (DDR-2). Only a small subset of myocardial fibroblasts was positive for DDR-2. Nevertheless, we detected more DDR-2-positive fibroblasts in the hearts of 70-d-old LP rats (18.36 \pm 1.51 DDR-2-positive fibroblasts per view in LP rats vs. $11.77 \pm 1.22 \mathrm{DDR}-2$-positive fibroblasts per view in NP rats, $P<0.01$ ). The costaining for vimentin and $\alpha$ SMA revealed that vimentin-positive fibroblasts were not coreactive with $\alpha$-SMA (Figure 6f), indicating that fibroblasts in the hearts of NP and LP rats were not activated myofibroblasts. a

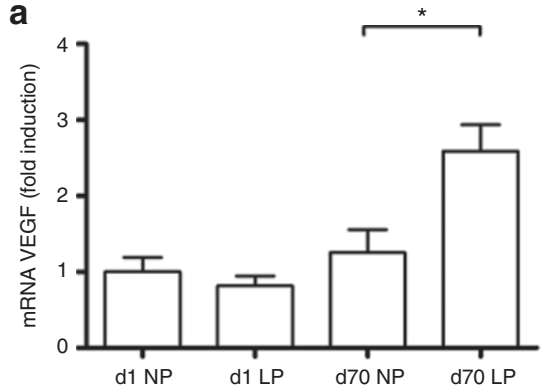

b

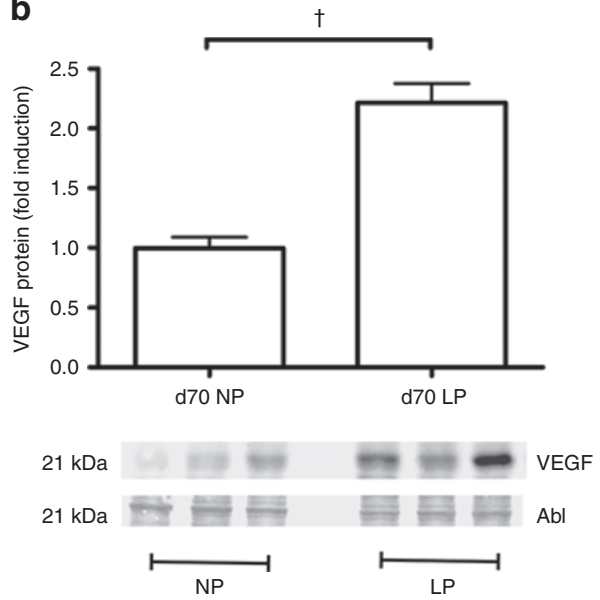

C

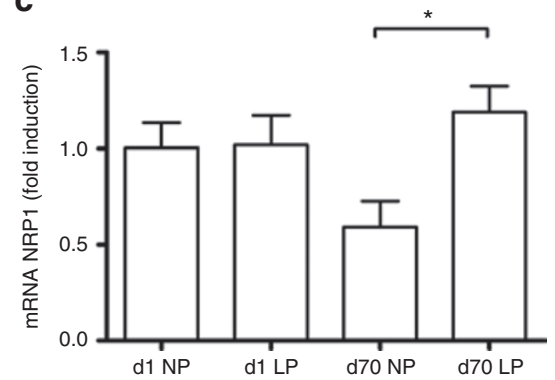

d

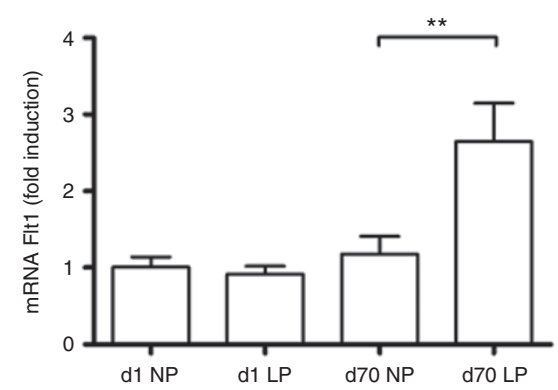

e

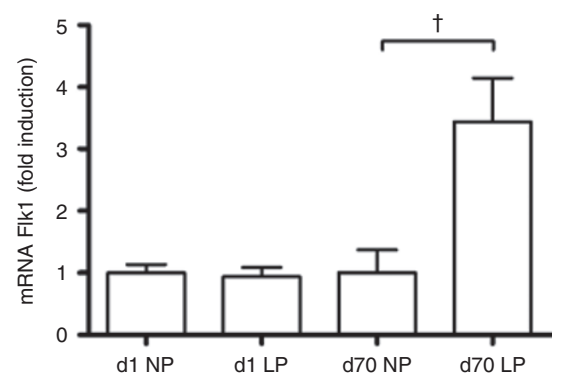

Figure 3. Myocardial expression of angiogenic factors. Expression of vascular endothelial growth factor (VEGF) (a) on the mRNA level and (b) on the protein level; mRNA expression levels of (c) the coreceptor neuropilin 1 (NRP1), (d) the VEGF receptor 1 (Flt1), and (e) the VEGF receptor 2 (Flk1) in rats at day 1 (d1) and at day 70 (d70) of life. Abl, amido black staining as a loading control. Data are means $\pm \mathrm{SEM},{ }^{*} P<0.05,{ }^{* *} P<0.01,{ }^{\dagger} P<0.001$. LP, rats with intrauterine growth restriction; NP, control rats. 
a

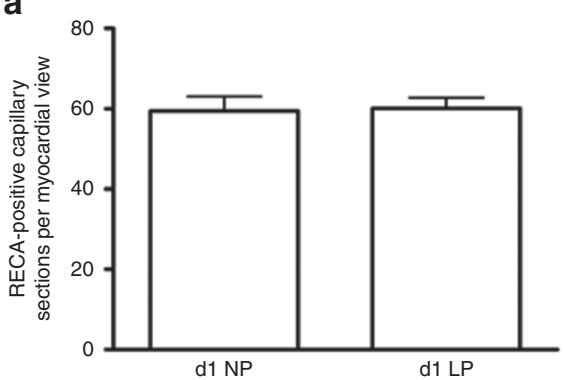

b

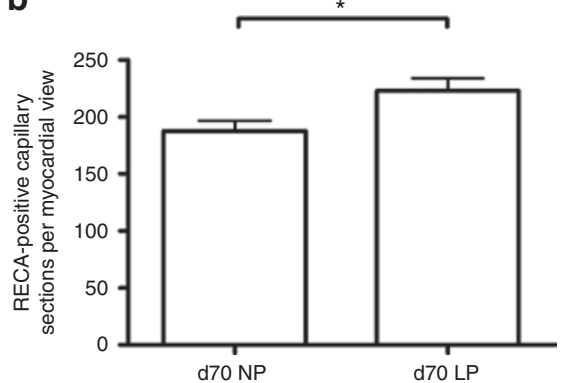

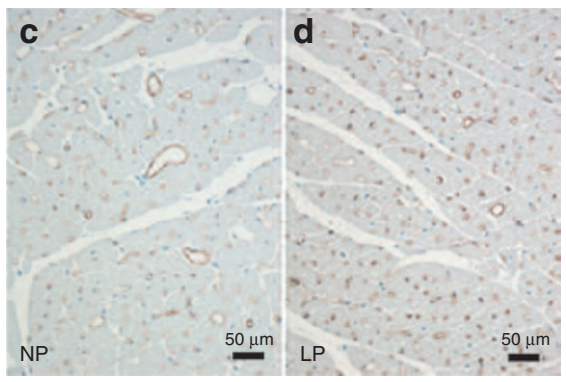

Figure 4. Evaluation of myocardial vascularization using immunohistochemistry for RECA as a marker for endothelial cells. (a) Quantification of vascular cross-sections stained with RECA in myocardial tissue of rats at day 1 (d1) of life. (b) Quantification of vascular cross-sections stained with RECA in myocardial tissue of rats at day 70 (d70) of life. (c,d) Representative photomicrographs of RECA stained myocardial tissue. Data are means \pm SEM. ${ }^{*} P<0.05$. LP, rats with intrauterine growth restriction; NP, control rats.

a

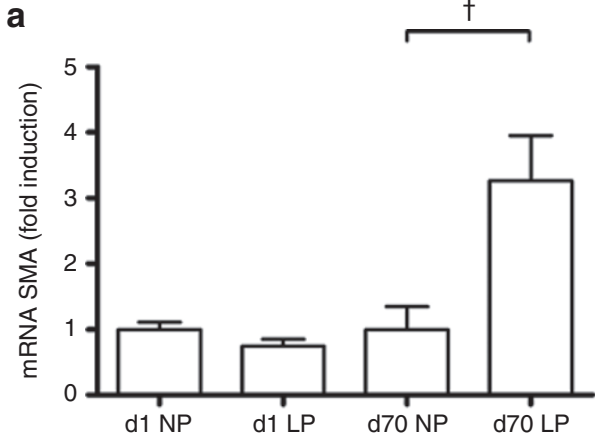

b
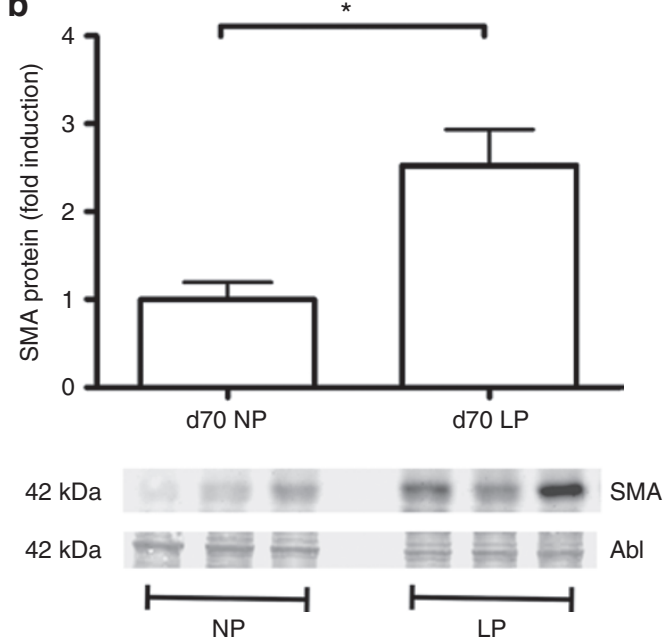

C

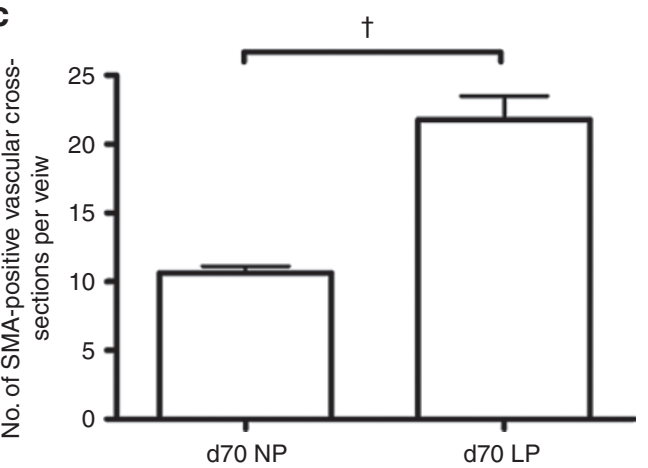

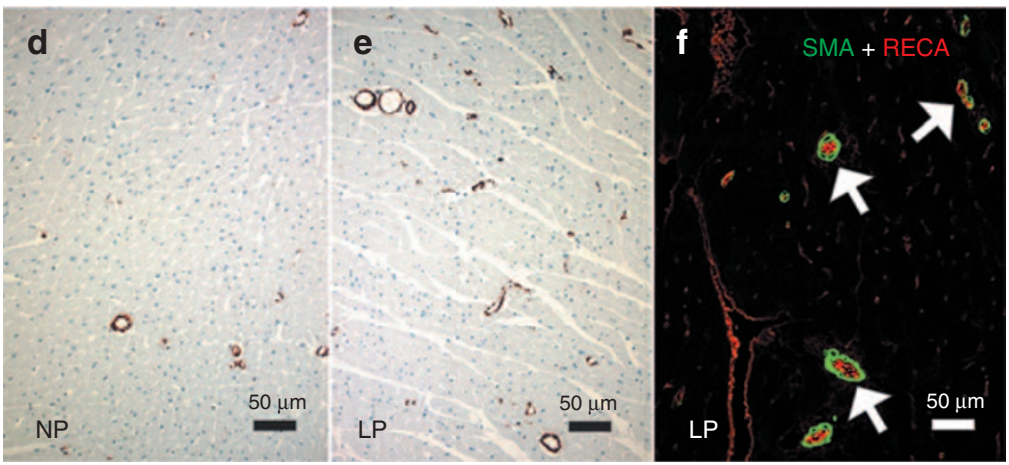

Figure 5. Evaluation of myocardial vascularization using $\alpha$-smooth muscle actin (SMA) as a marker for smooth muscle cells. (a) Myocardial mRNA expression levels of SMA in rats at day 1 (d1) and at day 70 (d70) of life. (b) Quantification of myocardial SMA protein content in rats at d70 of life. Abl, amido black staining as a loading control. (c) Immunohistochemical quantification of vascular cross-sections stained with SMA in myocardial tissue of 70-d-old rats (d70). (d,e) Representative photomicrographs SMA-stained myocardial tissue at d70 of life. (f) Double immunofluorescence for SMA (green) and RECA (red) in myocardial tissue at d70 of life confirming that SMA staining is associated with RECA (white arrows) and can thus be attributed to blood vessels. Original magnification $\times 240$. Data are means \pm SEM. ${ }^{*} P<0.05,{ }^{\dagger} P<0.001$. LP, rats with intrauterine growth restriction; NP, control rats.

\section{DISCUSSION}

The main finding of this study is that our animal model of IUGR leads to an early impaired myocardial function. Moreover, the reduced cardiac performance is accompanied by a dysregulation of sarcomeric components. Day 70 of life was chosen as end point for functional and molecular investigations because it corresponds to a human age of 5-6 y (11). For this age, cohort clinical studies could show significant alterations of myocardial structure and functionality after IUGR (6). Contrary to a number of other IUGR models, the impaired myocardial function in our study occurs independently from arterial hypertension and can therefore be considered as a primary IUGR-induced 


\section{Articles | Menendez-Castro et al.}
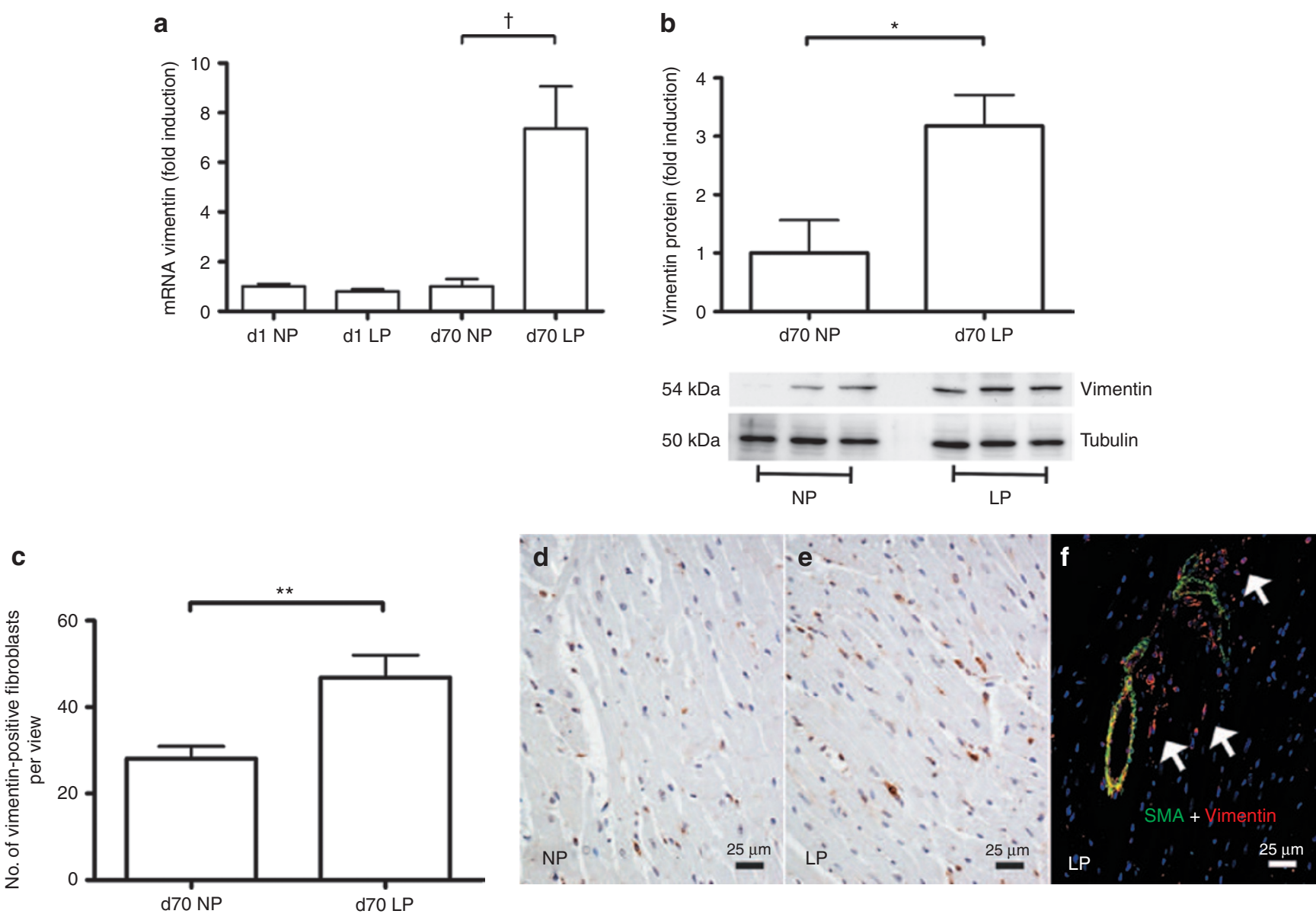

Figure 6. Evaluation of myocardial vimentin as a marker for fibroblasts. (a) Myocardial mRNA expression levels of vimentin in rats at day 1 (d1) and at day 70 (d70) of life. (b) Quantification of myocardial vimentin protein content in rats at d70 of life. Tubulin staining served as a loading control for western blot analysis. (c) Immunohistochemical quantification of vimentin-positive fibroblasts in myocardial tissue of rats at d70 of life. (d,e) Representative photomicrographs of vimentin-stained myocardial tissue at d70 of life. (f) Double immunofluorescence for vimentin (red) and $\alpha$-smooth muscle actin (SMA; green) in myocardial tissue at d70 of life confirming that vimentin-positive fibroblasts are not coreactive with $\alpha$-SMA (white arrows). Original magnification $\times 480$. Data are means $\pm \mathrm{SEM} .{ }^{*} P<0.05,{ }^{* *} P<0.01,{ }^{\dagger} P<0.001$. LP, rats with intrauterine growth restriction; NP, control rats.

effect $(10,12)$, which may favor the development of cardiac disease later in life. Concerning the relationship between arterial hypertension and IUGR induced by maternal protein restriction, differing reports can be found showing blood pressure elevation (13) or, alternatively, also unaffected blood pressures levels (14). This can partly be explained by different time points and methods of blood pressure assessment (e.g., telemetric blood pressure measurement vs. tail cuff plethysmography) as well as different protocols of maternal protein restriction (15).

Echocardiography in IUGR rats at day 70 of life revealed significantly higher values of LVEDD and LVESD with a consecutively reduced fractional shortening. Accompanied by a decreased ejection fraction and a reduction of left ventricular anteriolateral wall thickness, these data point to an impaired contractile function and indicate a more distensible myocardial structure in IUGR rats at this time point, which is supportive of the findings of Crispi et al. (6) in humans. Similar results were obtained in an IUGR model with increased LVEDD and reduced ejection fraction in juvenile IUGR rats (8). Using an in vivo catheterization technique, these authors detected a reduction in cardiac output, accompanied by decreased arterial blood pressure (8). At least a trend toward reduced cardiac output was also observed in another low protein model of IUGR (16). Lim et al. (7) studied cardiac function in IUGR rats at an older age and in contrast to Cheema et al. (8) found reduced LVEDD and normal ejection fraction. The reasons for this discrepancy remain unclear but may include different modes of low protein feeding (during pregnancy only vs. during pregnancy and lactation) and different time points investigated (8).

An impaired cardiac function is often accompanied by altered expression patterns of contractile myocardial components. For example, the expression of MHCs reflects compensatory mechanisms under increased myocardial load (17) as well as in manifest cardiomyopathy (18). Compared with $\alpha$-MHC, $\beta$-MHC is considered as the MHC isoform with a higher degree of efficiency (19). Assessing the expressional ratio of $\alpha-\mathrm{MHC} / \beta-\mathrm{MHC}$, we were able to show at least a tendency toward an overexpression of $\beta$-MHC in 70-d-old LP rats. This could support the hypothesis of a compensatory myocardial response to the affected myocardial performance post-IUGR. In another IUGR model using maternal low sodium diet, leading to manifest arterial hypertension in the offspring, Battista 
et al (12). did not observe changes of the expression of $\beta$-MHC in 12-wk-old female Sprague-Dawley IUGR rats. In contrast to our study, they could not detect any functional alterations in male IUGR offspring at all. This discrepancy might be explained by different underlying pathomechanisms in these two models of IUGR (low protein vs. low sodium). In contrast to the findings of Battista et al. (12), changes in myosin chain expression were not accompanied by elevated blood pressure in our model. The increased expression of myosin chains paralleling the impaired myocardial performance might reflect a very early compensatory mechanism to strengthen myocardial contractility after IUGR. This hypothesis is supported by other studies showing the cardioprotective effect of MHCs in myocardial pathologies (20).

Besides actin and myosin, titin is another important component of the myocardial sarcomeres. As a structural protein, it determines the passive elasticity of cardiomyocytes and thus secures the structural integrity of the myocardium under pressure and volume load (21). By alternative splicing, two main isoforms of titin, N2B and N2BA, differing in their elastic properties, occur postnatally in the mammalian myocardium. Compared with N2B, N2BA shows a higher extensibility and a reduced resistance to extension forces (22). In our study, we detected an increased expression of both $\mathrm{N} 2 \mathrm{~B}$ and N2BA in 70-d-old IUGR animals. Assessment of the expressional ratio of N2BA/N2B showed a significant increase in LP rats, indicating a relative overweight of N2BA in the myocardium of these animals. The somewhat stronger increase of N2BA expression compared with N2B in our IUGR rats together with the significantly elevated values of LVEDD and LVESD in these animals supports the hypothesis of a more distensible myocardium post-IUGR. According to this, an overexpression of N2BA could be found in the myocardium of patients with dilated cardiomyopathy (23).

Cardiac fibroblasts play a central role in maintaining myocardial structure and functionality. During cardiac injury, a differentiation of cardiac fibroblasts into myofibroblasts occurs, which supports tissue repair but also may contribute to cardiac fibrosis (24). In our study, we detected a higher number of cardiac fibroblasts in IUGR rats. Costaining for the fibroblast marker vimentin and $\alpha$-SMA as marker for myofibroblasts confirmed that the increased number of fibroblasts did not reflect an accumulation of myofibroblasts after IUGR. Nevertheless, an increased number of cardiac fibroblasts may be responsible for the altered myocardial matrix composition in IUGR rats observed in a previous study (10) and may thus alter myocardial elasticity.

The myocardium has the ability to adapt and to optimize its performance by alteration of its structure and functionality. In this context, it could be shown that markers of growth and differentiation were reactivated (25). One of them is ANP. During embryonal development, ANP is highly expressed in the atrium and the ventricle. After birth, only a reduced expression of ANP can be found in the healthy, unaffected mammalian heart (26). A postnatal resurgence of ventricular ANP expression can be found following unphysiological cardiac load and constitutes an early marker of structural and functional pathologies in the myocardium (27). In humans, increased concentrations of ANP in the umbilical vein blood of IUGR fetuses were described (28). Moreover, hypoxia of the myocardium was found to stimulate myocardial ANP expression (29). In our study, we detected a higher ventricular ANP expression and speculate that the more distensible myocardium after IUGR reaches a higher wall tension, which in turn could upregulate the expression of ANP.

Furthermore, $\beta$-adrenergic pathways are often activated to maintain an appropriate cardiac output (30), which could explain the augmented myocardial expression of the $\beta 1$ adrenergic receptor in our IUGR animals. On the other hand, chronic upregulation of $\beta$-adrenergic pathways leads to unfavorable hypertrophic changes of the myocardium in the long run (30). Thus, early activated cardioprotective mechanisms after IUGR can possibly also result in the development of cardiac disease.

Another important factor to regulate myocardial inotropy is the $\mathrm{Na} / \mathrm{K}$-ATPase. Animal studies revealed a positive correlation between $\mathrm{Na} / \mathrm{K}$-ATPase activity and myocardial inotropy, suggesting a cardioprotective function of $\mathrm{Na} / \mathrm{K}$-ATPase (31). We speculate that the overexpression of myocardial $\mathrm{Na} / \mathrm{K}$ ATPase, seen in our study, may represent a further compensatory mechanism.

To our surprise, we observed an increased vascular supply in the hearts of 70-d-old IUGR rats. Volume and pressure overload, along with a reduced coronary perfusion, can lead to ischemia and hypoxia (32). We detected a higher myocardial expression of the HIF-1 target genes, IGFBP-3, HO- 1 , and HIG-2, indicating myocardial hypoxia in IUGR animals at this time point. Assessing neoangiogenic factors, we could also show higher levels of VEGF and its receptors Flt1, Flk1, and NRP1 in these animals. Neoangiogenesis is frequently associated with myocardial hypertrophy (33). In our study, relative heart weights of 70-d-old LP and NP rats are not different, which is not supportive of a hypertrophic myocardial remodeling in IUGR animals as a potential neoangiogenic trigger. We hypothesize that in our model of IUGR the increased distensibility of the myocardium detected by echocardiography might lead to higher mechanical strain and subsequently to hypoxic induction of vascular growth. In this context, it could be shown in vitro that mechanical stress to cardiomyocytes induces VEGF (34). Accordingly, Tintu et al. (5) described the phenotype of a dilated cardiomyopathy and an increased expression of VEGF in chick embryos after hypoxia-induced IUGR. In contrast to our findings, Lim et al. (7) did not detect any changes in myocardial capillarization in female IUGR rats. These conflicting data might arise from gender differences, different modes of low protein feeding, or different time points investigated.

Taken together, we detected impaired cardiac function and echocardiographic signs of a more distensible myocardium post-IUGR in the absence of arterial hypertension. These observations are accompanied by a dysregulation of sarcomeric components and an increase in myocardial 
Table 3. Primer pairs used for quantitative RT-PCR analysis

\begin{tabular}{|c|c|c|}
\hline & Forward & Reverse \\
\hline Adrb-1 & 5'- ATCGTAGTGGGCAACGTGTTG -3' & 5'- AGGGACATGATGAAGAGGTTGGT - $3^{\prime}$ \\
\hline ANP & 5'-TTCAAGAACCTGCTAGACCA - $3^{\prime}$ & 5'-GCTCCAATCCTGTCAATCCT-3' \\
\hline HO-1 & 5'-GCCTGCTAGCCTGGTTCAAGA -3' & 5'-GAGTGTGAGGACCCATCGCA -3' \\
\hline IGFBP-3 & 5'-GTGCGCTTAGTTCTCCGGATGAC - $3^{\prime}$ & 5'-CCAGGGTCTCCAACAAGGAAGATG -3' \\
\hline$\beta-\mathrm{MHC}$ & $5^{\prime}-\mathrm{TCAAAAGGGCCTGAATGAAGA}-3^{\prime}$ & 5'-GGGCTTCACAGGCATCCTTAG - $3^{\prime}$ \\
\hline $\mathrm{N} 2 \mathrm{~B}$ & 5'-CCAACGAGTATGGCAGTGTCA -3' & 5'-TGGGTTCAGGCAGTAATTTGC - $3^{\prime}$ \\
\hline N2BA & 5'-CGGCAGAGCTCAGAATCGA -3' & 5'- GTCAAAGGACACTTCACACTCAAAA -3' \\
\hline $\mathrm{Na} / \mathrm{K}$-ATPase & 5'-TGGAGACTTACCCTCTGACGATG - $3^{\prime}$ & 5'- GGCTAGTGGGAAAGATTTGTGC - $3^{\prime}$ \\
\hline NRP1 & $5^{\prime}$-TGGAGCTACTGGGCTGTGAA-3' & 5'-GTGTCGTGGGTCCAGCTGT -3' \\
\hline VEGF-R1 flt & 5'- CGACACTCTTTTGGCTCCTTCTAAC - $3^{\prime}$ & 5'-TGACAGGTAGTCCGTCTTTACTTCG -3' \\
\hline VEGF-R2 flk & $5^{\prime}$ - CCACCCCAGAAATGTACCAAAC - $3^{\prime}$ & 5'- AAAACGCGGGTCTCTGGTT -3' \\
\hline Vimentin & $5^{\prime}$ - AAACGAATACCGGAGACA - $3^{\prime}$ & 5'-CCAGGGACTCATTAGTGCCTTT -3' \\
\hline $18 \mathrm{~S}$ & 5'-TTGATTAAGTCCCTGCCCTTTGT -3' & 5'-CGATCCGAGGGCCTCACTA-3' \\
\hline
\end{tabular}

$\alpha$-MHC, $\alpha$-myosin heavy chain; $\alpha$-SMA, $\alpha$-smooth muscle actin; $\beta$-myosin heavy chain; Adrb-1, $\beta$-adrenergic receptor 1 ; ANP, atrial natriuretic peptide; HO-1, heme oxygenase 1 ; NRP1, neuropilin 1; VEGF, vascular endothelial growth factor.

vascularization. The fact that differences between IUGR animals and controls revealed only at day 70 of life, not at day 1 of life, is supported by clinical observations: it is not unusual that congenital myocardial pathologies unmask postnatally with some delay because of the physiologically higher ventricular load appearing after birth compared to intrauterine hemodynamics (35). The observed alterations might in part reflect a compensatory response to a reduced myocardial performance or to myocardial hypoxia. On the other hand, it seems conceivable that an early dysregulation of contractile components, like the titin isoforms, with a relative overexpression of the more distensible isoform, might impair myocardial function and thus pave the way for the development of cardiac disease later in life.

\section{METHODS}

\section{Animal Model}

All procedures performed on animals conform to the Guide for the Care and Use of Laboratory Animals published by the US National Institutes of Health (NIH Publication No. 85-23, revised 1996) and were approved by local government authorities (Regierung von Mittelfranken, AZ \#54-2531.31-12/06). As described previously, IUGR was induced using the rat model of maternal protein restriction during pregnancy (10). After spontaneous delivery, body weights of the offspring were assessed, and the litters were reduced to six male pups per dam as described (36) to achieve equal lactation conditions. The offspring of the mothers fed a diet with normal protein content were termed NP, the offspring of the mother with low protein diet were termed LP. Surplus male pups were killed by decapitation, and their hearts were used for histological and molecular evaluation. During lactation, rat mothers were fed standard chow. The offspring was nursed by their mothers until weaned at day 21 to standard chow. We used hearts from 24 male animals from six litters per group at day 1 of life and hearts from 20 animals from five litters per group at day 70 of life.

\section{Echocardiography}

As described previously (37), echocardiography was performed the day before sacrifice of 70 -d-old rats ( 10 per group, randomly assigned) under mild isoflurane anesthesia $\left(\mathrm{O}_{2}\right.$ flow of $1 \mathrm{l} / \mathrm{min}, 2 \%$ isoflurane) by one experienced examiner using a linear transducer of $13 \mathrm{MHz}$ frequency on a Acuson CV 70 Cardiovascular 4.0 (Siemens, Munich, Germany) in a blinded manner. Data were obtained by B-mode in the left long and short parasternal axes and by M-mode in the short left parasternal axis at average heart rates of $325 \mathrm{bpm}$. The values of LVESD, LVEDD, left ventricular anteriolateral wall thickness, and left ventricular posterior wall thickness were assessed by M-mode tracings. The percentage of left ventricular fractional shortening was calculated as [(LVEDD - LVESD) / LVEDD] × 100 .

\section{Blood Pressure Measurements}

Intraarterial blood pressure measurements were performed as described (10) in all rats of the experimental groups. At the day of sacrifice, catheters were implanted in the right femoral artery and tunneled subcutaneously. After a recovery phase of $2 \mathrm{~h}$, mean arterial blood pressure was recorded by a polygraph (Hellige, Freiburg, Germany) in conscious animals for $30 \mathrm{~min}$.

\section{Tissue Preparation}

At day 70 of life, animals were killed by retrograde perfusion in deep anesthesia (ketamine $100 \mathrm{mg} / \mathrm{kg}$ body weight and midazolam $5 \mathrm{mg} / \mathrm{kg}$ body weight intraperitoneally). Hearts were removed and weighted. A 3-mm-thick apical part of left ventricular tissue was snap frozen in liquid nitrogen. Another 3-mm-thick specimen of left ventricular tissue was fixed in methyl carnoy solution and imbedded in paraffin for immunohistochemistry. Two-micrometer sections were cut, using a Leitz microtome (Leica Instruments, Wetzlar, Germany).

\section{Immunohistochemistry}

Staining of paraffin sections was performed as previously described (38). To detect blood vessels in myocardial tissue, sections were stained with an anti-rat endothelial cell antigen (RECA, AbD Serotec, Düsseldorf, Germany) antibody in a dilution of 1:20 and an antihuman $\alpha$-SMA (DAKO, Hamburg, Germany) antibody in a dilution of 1:50. To detect myocardial fibroblasts, an anti-rat vimentin antibody (Progen, Heidelberg, Germany) was applied in a dilution of 
1:100. Stained myocardial vessels or fibroblasts were counted in 5-10 medium-power views per section. Only myocardial views containing vessels smaller than $50 \mu \mathrm{m}$ in diameter were counted. Furthermore, a second antibody staining fibroblasts, a DDR-2 antibody, was used in a dilution of 1:400 (Santa Cruz Biotechnology, Heidelberg, Germany) as previously described (39). DDR-2-positive cells were counted in five low-power myocardial views. Double immunofluorescence stainings for RECA and $\alpha$-SMA were performed by detection of RECA with a goat anti-mouse Alexa555 secondary antibody and subsequently of $\alpha$-SMA with a donkey anti-mouse Alexa488 secondary antibody (both Abcam, Cambridge, UK). Double immunofluorescence stainings for vimentin and $\alpha$-SMA were performed by simultaneous incubation of sections with both primary antibodies, followed by simultaneous incubation with the secondary antibodies goat anti-guinea pig Alexa555 and donkey anti-mouse Alexa488 (1:500, Abcam).

\section{Western Blot Analysis}

Frozen myocardial tissue was processed, and electrophoresis was performed as described previously (10) The gels were electroblotted onto polyvinylidene difluoride membranes (Hybond-P, GE Amersham, Munich, Germany), blocked with Rotiblock (Roth, Karlsruhe, Germany) for $1 \mathrm{~h}$, and incubated overnight with a primary antibody. Primary antibodies were as follows: Adrb-1 (Abcam, 1:1000), ANP (Santa Cruz Biotechnology, 1:200), $\alpha$-SMA (DAKO, 1:5000), VEGF (Santa Cruz Biotechnology, 1:2000), and vimentin (Progen, 1:1000). Protein bands were visualized with secondary horseradish peroxidase-conjugated IgG antibodies (Santa Cruz Biotechnology, 1:50000), using the ECL system (GE Amersham, Freiburg, Germany). Blots were quantified using a luminescent imager (LAS-1000, Fujifilm, Berlin, Germany) and Aida 2.1 image analysis software (Raytest, Berlin, Germany). Loading of the blot was quantified by reprobing with an antibody to tubulin (Sigma, Taufkirchen, Germany, 1:10000) or by amido black staining.

\section{Real-Time RT-PCR}

Total RNA from myocardial tissue was extracted, and first-strand cDNA was synthesized as described previously (10). RT-products were diluted 1:1 with $\mathrm{dH}_{2} \mathrm{O}$ before PCR procedure. PCR was performed with an ABI PRISM 7000 Sequence Detector System and SYBR Green reagents (Applied Biosystems, Foster City, CA) according to the manufacturer's instructions. Primers used for real-time RT-PCR are listed in Table 3. Primer pairs were designed using Primer Express software (Perkin Elmer, Foster City, CA). All samples were run in triplicates. Relative quantities and change of mRNA expression of target genes were calculated after normalization to $18 \mathrm{~S}$ rRNA as a housekeeping gene and with the $\Delta \Delta C_{\mathrm{T}}$ method as specified by the manufacturer (Applied Biosystems).

\section{Analysis of Data}

All data are expressed as means \pm SEM. Student's $t$-test was performed to assess the differences between groups at day 70 of life, one-way ANOVA followed by a post hoc Bonferroni test was used to assess the differences of real-time PCR expression analyses using GraphPad Prism 5 (GraphdPad Software, La Jolla, CA). Results were considered significant when the probability of error ( $P$ value) was less than 0.05 .

\section{ACKNOWLEDGMENTS}

We gratefully acknowledge the expert technical assistance of Ilona Winterfeld and Miroslava Kupraszewicz-Hutzler.

\section{STATEMENT OF FINANCIAL SUPPORT}

This study was supported by an Erlanger Leistungsbezogene Anschubfinanzierung und Nachwuchsfoerderung (ELAN) grant from the University Hospital of Erlangen-Nürnberg to C.M.-C. and by a grant from the "Deutsche Stiftung für Herzforschung" to O.T.

Disclosure: There are no competing financial, personal, or professional interests for any of the authors.

\section{ADDITIONAL AUTHOR INFORMATION}

C.M.-C., A.H., and K.F.H. designed the study and contributed to the manuscript. W.R. contributed to the design of the study and critically revised the manuscript. F.F., R.W., and N.C. collected, analyzed, and interpreted the data. O.T. contributed to the design of the study and performed the echocardiography. C.M.-C. interpreted the data and drafted the manuscript.

\section{REFERENCES}

1. Barker DJ. In utero programming of cardiovascular disease. Theriogenology 2000;53:555-74.

2. McMillen IC, Robinson JS. Developmental origins of the metabolic syndrome: prediction, plasticity, and programming. Physiol Rev 2005; 85:571-633.

3. Barker DJ. In utero programming of chronic disease. Clin Sci (Lond) 1998;95:115-28.

4. Corstius HB, Zimanyi MA, Maka N, et al. Effect of intrauterine growth restriction on the number of cardiomyocytes in rat hearts. Pediatr Res 2005;57:796-800.

5. Tintu A, Rouwet E, Verlohren S, et al. Hypoxia induces dilated cardiomyopathy in the chick embryo: mechanism, intervention, and long-term consequences. PLoS One 2009;4:e5155.

6. Crispi F, Bijnens B, Figueras F, et al. Fetal growth restriction results in remodeled and less efficient hearts in children. Circulation 2010;121:2427-36.

7. Lim K, Lombardo P, Schneider-Kolsky M, Black MJ. Intrauterine growth restriction coupled with hyperglycemia: effects on cardiac structure in adult rats. Pediatr Res 2012;72:344-51.

8. Cheema KK, Dent MR, Saini HK, Aroutiounova N, Tappia PS. Prenatal exposure to maternal undernutrition induces adult cardiac dysfunction. Br J Nutr 2005;93:471-7.

9. Vehaskari VM, Woods LL. Prenatal programming of hypertension: lessons from experimental models. J Am Soc Nephrol 2005;16:2545-56.

10. Menendez-Castro C, Fahlbusch F, Cordasic N, et al. Early and late postnatal myocardial and vascular changes in a protein restriction rat model of intrauterine growth restriction. PLoS One 2011; 6:e20369.

11. Andreollo NA, Santos EF, Araújo MR, Lopes LR. Rat's age versus human's age: what is the relationship? Arq Bras Cir Dig 2012;25:49-51.

12. Battista MC, Calvo E, Chorvatova A, Comte B, Corbeil J, Brochu M. Intrauterine growth restriction and the programming of left ventricular remodelling in female rats. J Physiol 2005;565(Pt 1):197-205.

13. Langley-Evans SC, Welham SJ, Jackson AA. Fetal exposure to a maternal low protein diet impairs nephrogenesis and promotes hypertension in the rat. Life Sci 1999;64:965-74.

14. Hoppe CC, Evans RG, Moritz KM, et al. Combined prenatal and postnatal protein restriction influences adult kidney structure, function, and arterial pressure. Am J Physiol Regul Integr Comp Physiol 2007;292:R462-9.

15. Zohdi V, Sutherland MR, Lim K, Gubhaju L, Zimanyi MA, Black MJ. Low birth weight due to intrauterine growth restriction and/or preterm birth: effects on nephron number and long-term renal health. Int J Nephrol 2012;2012:136942.

16. Zohdi V, Jane Black M, Pearson JT. Elevated vascular resistance and afterload reduce the cardiac output response to dobutamine in early growthrestricted rats in adulthood. Br J Nutr 2011;106:1374-82.

17. Gupta M, Zak R. Reversibility of load-induced changes in myosin heavy chain gene expression. Am J Physiol 1992;262(3 Pt 2):R346-9.

18. Abraham WT, Gilbert EM, Lowes BD, et al. Coordinate changes in Myosin heavy chain isoform gene expression are selectively associated with alterations in dilated cardiomyopathy phenotype. Mol Med 2002;8:750-60.

19. Sugiura S, Kobayakawa N, Fujita H, et al. Comparison of unitary displacements and forces between 2 cardiac myosin isoforms by the optical trap technique: molecular basis for cardiac adaptation. Circ Res 1998;82:1029-34.

20. James J, Martin L, Krenz M, et al. Forced expression of alpha-myosin heavy chain in the rabbit ventricle results in cardioprotection under cardiomyopathic conditions. Circulation 2005;111:2339-46.

21. Cazorla O, Freiburg A, Helmes M, et al. Differential expression of cardiac titin isoforms and modulation of cellular stiffness. Circ Res 2000;86:59-67. 


\section{Articles $\mid$ Menendez-Castro et al.}

22. Neagoe C, Opitz CA, Makarenko I, Linke WA. Gigantic variety: expression patterns of titin isoforms in striated muscles and consequences for myofibrillar passive stiffness. J Muscle Res Cell Motil 2003;24:175-89.

23. Makarenko I, Opitz CA, Leake MC, et al. Passive stiffness changes caused by upregulation of compliant titin isoforms in human dilated cardiomyopathy hearts. Circ Res 2004;95:708-16.

24. Fan D, Takawale A, Lee J, Kassiri Z. Cardiac fibroblasts, fibrosis and extracellular matrix remodeling in heart disease. Fibrogenesis Tissue Repair 2012;5:15.

25. Izumo S, Nadal-Ginard B, Mahdavi V. Protooncogene induction and reprogramming of cardiac gene expression produced by pressure overload. Proc Natl Acad Sci USA 1988;85:339-43.

26. Zeller R, Bloch KD, Williams BS, Arceci RJ, Seidman CE. Localized expression of the atrial natriuretic factor gene during cardiac embryogenesis. Genes Dev 1987;1:693-8.

27. Adachi S, Ito H, Ohta Y, et al. Distribution of mRNAs for natriuretic peptides in RV hypertrophy after pulmonary arterial banding. Am J Physiol 1995;268(1 Pt 2):H162-9.

28. Bahlmann F, Krummenauer F, Spahn S, Gallinat R, Kampmann C. Natriuretic peptide levels in intrauterine growth-restricted fetuses with absent and reversed end-diastolic flow of the umbilical artery in relation to ductus venosus flow velocities. J Perinat Med 2011;39:529-37.

29. Klinger JR, Pietras L, Warburton R, Hill NS. Reduced oxygen tension increases atrial natriuretic peptide release from atrial cardiocytes. Exp Biol Med (Maywood) 2001;226:847-53.

30. Port JD, Bristow MR. Altered beta-adrenergic receptor gene regulation and signaling in chronic heart failure. J Mol Cell Cardiol 2001;33:887-905.
31. Xu KY, Takimoto E, Fedarko NS. Activation of $(\mathrm{Na}++\mathrm{K}+)$-ATPase induces positive inotropy in intact mouse heart in vivo. Biochem Biophys Res Commun 2006;349:582-7.

32. Jeremy RW, Fletcher PJ, Thompson J. Coronary pressure-flow relations in hypertensive left ventricular hypertrophy. Comparison of intact autoregulation with physiological and pharmacological vasodilation in the dog. Circ Res 1989;65:224-36.

33. Tomanek RJ. Response of the coronary vasculature to myocardial hypertrophy. J Am Coll Cardiol 1990;15:528-33.

34. Leychenko A, Konorev E, Jijiwa M, Matter ML. Stretch-induced hypertrophy activates NFkB-mediated VEGF secretion in adult cardiomyocytes. PLoS One 2011;6:e29055.

35. Arola A, Tuominen J, Ruuskanen O, Jokinen E. Idiopathic dilated cardiomyopathy in children: prognostic indicators and outcome. Pediatrics 1998;101(3 Pt 1):369-76.

36. Plank C, Ostreicher I, Hartner A, et al. Intrauterine growth retardation aggravates the course of acute mesangioproliferative glomerulonephritis in the rat. Kidney Int 2006;70:1974-82.

37. Teekakirikul P, Eminaga S, Toka $\mathrm{O}$, et al. Cardiac fibrosis in mice with hypertrophic cardiomyopathy is mediated by non-myocyte proliferation and requires Tgf- $\beta$. J Clin Invest 2010;120:3520-9.

38. Haas CS, Amann K, Schittny J, Blaser B, Müller U, Hartner A. Glomerular and renal vascular structural changes in alpha8 integrin-deficient mice. J Am Soc Nephrol 2003;14:2288-96.

39. Hartner A, Cordasic N, Rascher W, Hilgers KF. Deletion of the alpha8 integrin gene does not protect mice from myocardial fibrosis in DOCA hypertension. Am J Hypertens 2009;22:92-9. 